\title{
Dinámicas de publicación global
}

\section{Literaturas del Caribe}

Las dinámicas del Global Publishing han experimentado relevantes cambios desde la década de 1960, pero muy especialmente en los últimos treinta años, a los que me referiré en este trabajo. Las multinacionales mediáticas, como se sabe, determinan el funcionamiento de amplios sectores del mercado del libro. André Schiffrin, ex editor de Pantheon-Books y figura sumamente importante sobre todo en la canonización de las literaturas latinoamericanas en Estados Unidos, tituló su ensayo The Business of Books: How International Conglomerates Took Over Publishing and Changed the Way We Read, que publicó en el año 2000, después de que la multinacional Bertelsmann comprara el grupo editorial al que pertenecía Pantheon. Otro problema tratado desde varias perspectivas es el creciente número de títulos que aparecen cada año. Ann Steiner informa que en el año 2009 se publicaron en Gran Bretaña 120000 títulos, 90000 en Alemania, 70000 en la India, 120000 en Rusia, 275000 en Estados Unidos y unos 150000 en China. Ann Steiner comenta al respecto: "Export, as these figures indicate, is necessary, and particularly the British, American, Spanish, French and German book trades depend on a large international market. Former colonies, trade bonds, media ownership, and other kinds of channels link the publishing industries in different countries" (2011: 319). De este modo, ciertas redes establecidas, tanto políticas como económicas, son empleadas como catalizadoras del mercado internacional del libro. ¿Qué significan pues los desafíos de la globalización para el ámbito literario?

Partiendo de la perspectiva sociológica de Pierre Bourdieu, Gisèle Sapiro se ocupa de la producción y la circulación literarias en un ámbito literario transnacional y, más exactamente, de los factores políticos, económicos, culturales y sociales que impulsan o impiden la circulación de la literatura mundial con independencia de su valor intrínseco (véase, por ejemplo, Sapiro/Pacouret/Picaud 2015; Sapiro 2016: 81). En el marco de los mecanismos de la industria editorial, Sapiro nos llama la atención acerca de los obstáculos que se interponen en el camino de la distribución internacional de la literatura, como la ausencia de redes de distribución, el alto costo de los derechos autorales o el criterio de la rentabilidad. Según ella, la traducción literaria tiene siempre, también, una significación política, ya que se corresponde con las relaciones de poder entre

Gesine Müller, Universität zu Köln

Ә Open Access. () 2021 Gesine Müller, published by De Gruyter. (cc) BY-NC-ND This work is licensed under a Creative Commons Attribution-NonCommercial-NoDerivatives 4.0 International License.

https://doi.org/10.1515/9783110713015-008 
los países: "For a nation-state, exporting its literature in translation is a sign of its symbolic recognition on the international scene” (Sapiro 2016: 84). Los efectos de la globalización en la difusión de la literatura traducida son vistos por esta autora como arma de doble filo: "Though the dynamics of globalization stimulated the local book industry in many countries and fostered cultural exchange through translation (the number of translations in the world increased by $50 \%$ between 1980 and 2000 according to the Index Translationum), the concentration process has had a negative impact on cultural diversity” (Sapiro 2016: 87). Este proceso está relacionado con el hecho de que los editores, partiendo de consideraciones relacionadas con las ventajas de operaciones seguras, tienden a comprar los derechos de aquellos libros que ya han sido adquiridos por otras editoriales. Ello tiene como consecuencia que solo circulan ciertos libros, con lo cual el mercado literario masivo se va volviendo cada vez más uniforme y monocultural.

No obstante, hay tendencias contrarias en el negocio editorial que no se basan en motivos financieros o ideológicos. El hecho de que aumente el número de traducciones de algunos idiomas periféricos, se explica, según Sapiro, debido a la suposición de que la literatura puede informar acerca de las culturas y las costumbres de un país (Sapiro 2016: 90). En el caso de esos criterios de selección entran a desempeñar un papel, sobre todo, determinados criterios simbólicos como el prestigio o el reconocimiento en el ámbito literario:

The publisher plays a major role by transferring its symbolic capital - encapsulated in its brand name (like a fashion designer's signature) - to the single work and to the author. The credit of the publisher is thus bequeathed to the authors he chooses to publish after a selection process.

There is, of course, a circularity in the dynamics of symbolic capital: a publisher partly accumulates symbolic capital by publishing authors that get attention through critical reception and prizes (Sapiro 2017: 82).

Para Sapiro, la política cultural internacional de la UNESCO, muy especialmente por la vía de los programas de subvenciones y los premios literarios, resultó decisiva para la paulatina inclusión de autores y autoras asiáticos o latinoamericanos en las décadas de 1960 y 1970, así como para la inclusión en el canon, a partir de los años 90, de autores y autoras postcoloniales y de mujeres. No obstante, la condición periférica de un idioma literario y de un ámbito cultural sigue contando con un menor reconocimiento en el marco de la literatura mundial (Sapiro 2016: 91ss.).

Con este planteamiento de fondo, las literaturas del Caribe anglófono, hispanófono o francófono jalonan un fascinante punto de partida para poner en perspectiva las investigaciones de los procesos de selección de literatura mundial de las editoriales con una actividad global. A la dinámica transfronteriza 
de las geopolíticas del Caribe corresponden ciertos métodos literarios que moldean y anticipan estéticamente la creación de redes transculturales (véase Müller/ Neumann: en prensa). Las literaturas del Caribe aportan un enorme potencial y son muy aptas para cuestionar los conceptos desfasados sobre la literatura mundial y llevar adelante concepciones innovadoras, lo mismo en los estudios culturales que en lo que atañe concretamente a la práctica del mundo de la literatura en la fase actual de globalización acelerada. Ello puede comprobarse, no en última instancia, si echamos una ojeada a los actuales programas de las grandes editoriales literarias de Europa y Estados Unidos, las cuales, por regla general, se esfuerzan por integrar en sus programas de publicaciones a autores y autoras sin residencia fija, autores que ya no pueden clasificarse dentro de una literatura nacional y combinan los patrones de movilidad transnacional como las innovadoras formas de expresión idiomática de un espacio “intermedio”. En el mercado del libro alemán, lo sintomático en este sentido lo constituye un aspecto planteado por el editor de Hanser, Jo Lendle, con motivo de la mesa redonda titulada Kölner Gespräche zur Weltliteratur [Diálogos colonienses sobre la Literatura Mundial], celebrado en el año 2018: "Cuando repaso lo que hemos hecho en Hanser en los últimos años y lo que vamos a hacer en los años siguientes, veo que se trata casi exclusivamente de autores que se encuentran en algún espacio intermedio"1.

Conocemos, en el Caribe anglófono, una tradición de literatura mundial que responde a los criterios del no asentamiento y el nomadismo, de la puesta en escena de movimiento en sustitución de las lógicas del centro y la periferia, y de un énfasis de lo particular, a menudo incluso de lo archipélico. Baste pensar en la epopeya Omeros, del escritor de Santa Lucía Derek Walcott, publicada en 1990, una obra "en muchos sentidos [. . .] paradigmática de la literatura mundial, y no tanto por su traducción a otros idiomas como por su consumación literaria de historias de transferencia y transformación que la convierten en un espacio multiestratificado de experimentación de una conciencia universal compartida, pero arraigada en lo local” (Müller/Neumann: en prensa). En el contexto de las lógicas de la literatura mundial y de los procesos de canonización, desempeña un papel decisivo el hecho de que los textos no tengan que ser traducidos a priori al que sigue siendo el idioma más importante de los procesos globales de circulación, es decir, el inglés, motivo por el cual el Caribe anglófono ocupa en este trabajo una posición especial. Los textos dignos de mención que han sido canonizados con éxito como literatura mundial son: The Arrivants (1967-1973), de Edward Kamau Brathwaite, Wide Sargasso Sea (1966),

1 Véase el video de esta mesa redonda en <https://youtu.be/8_4SoJ-PiH4>. 
de Jean Rhys, The Enigma of Arrival (1987), de V.S. Naipaul y In Another Place, Not Here (1996), de Dionne Brand.

También en lo concerniente a la traducción a otros idiomas, el inglés es, como sabemos, la lengua líder: en Alemania, por ejemplo, el porcentaje de libros traducidos entre 2007 y 2015 alcanzó cifras de entre 67\% y un 75\%, mientras que únicamente alrededor de un $16 \%$ de las traducciones de obras literarias no fueron realizadas del inglés o del francés ${ }^{2}$.

Otro factor no tan directamente visible para esta historia de éxitos de los textos literarios en lengua inglesa provenientes del Caribe reside en la aparición relativamente temprana de ciertas tendencias de desterritorialización en la formación del canon en Gran Bretaña o en Estados Unidos. No es casual que las autoras y autores que publicaron en el año 2007, en el periódico Le Monde, el varias veces citado manifiesto "Pour une 'littérature-monde' en français", con el cual dieron rienda suelta a su insatisfacción por la continuidad de las estructuras coloniales y exigieron la presencia de una literatura mundial de habla francesa “abierta al mundo y transnacional” (Barbery et al. 2007), pusieran como ejemplo el ámbito angloparlante. Cuando se trata de disolver el "pacto exclusivo con la nación” (Barbery et al. 2007), en el que la lengua francesa sigue girando, en opinión de esos autores y esas autoras, en torno a Maryse Condé, Édouard Glissant, Tahar Ben Jelloun, Dany Laferrière y muchos otros, ellos hacen referencia a su función modélica:

Combien d'écrivains de langue française, prix eux aussi entre deux ou plusieurs cultures, se sont interrogés alors sur cette étrange disparité qui les reléguait sur les marges, eux 'francophones', variante exotique tout juste tolérée, tandis que les enfants de l'ex-Empire britannique prenaient, en toute légitimité, possession des lettres anglaises? (Barbery et al. 2007).

En los años setenta, en Estados Unidos, se criticó con dureza la escasa consideración de las minorías sociales en el otorgamiento de premios literarios, lo mismo en lo relativo al premio Pulitzer que a la práctica de concesión de premios del National Book Award, hasta que el autor Ishmael Reed creó en 1980 los American Book Awards, que desde entonces son patrocinados por la Fundación Before Columbus. En un texto programático, reza en su página web:

The American Book Awards Program respects and honors excellence in American literature without restriction or bias with regard to race, sex, creed, cultural origin, size of press or ad budget, or even genre. [. . . ] The winners would not [be] selected by any set quota for diversity (nor would "mainstream white anglo male" authors be excluded), because diversity

2 Datos mencionados por Nicole Witt de la agencia literaria Witt Mertin con motivo de la mesa redonda Kölner Gespräche zur Weltliteratur en 2016. Véase <https://www.youtube.com/watch? $\mathrm{v}=\mathrm{xD}$ 5z4PJJLCQ $>$. 
happens naturally. [. . .] The only criteria would be outstanding contribution to American literature in the opinion of the judges (<http://www.beforecolumbusfoundation.com/ about/>).

Una contribución muy importante a un movimiento correspondiente en Estados Unidos la realizaron autores de Cuba y Puerto Rico, especialmente, también después de que, en 1990 - por señalar una fecha relevante- se otorgara por primera vez el Premio Pulitzer a un autor con antecedentes latinoamericanos. Oscar Hijuelos, nacido en 1951 en la ciudad de Nueva York, como hijo de inmigrantes cubanos, ganó el premio por su novela The Mambo Kings Play Songs of Love, publicada por Pantheon Books en 1989, con la cual obtuvo también una nominación al National Book Award y al National Book Critics Circle Prize. En general, en lo que atañe a los autores y autoras del Caribe en el mercado del libro estadounidense o británico, es posible constatar una canonización más temprana que la de los autores franco-caribeños en el mundillo literario francés.

Entre los pocos autores del ámbito francófono e hispanoparlante acogidos como literatura mundial, cabe mencionar al Premio Nobel (1960) Saint-John Perse - con sus poemas inspirados en la poesía moderna francesa-, el autor cubano Alejo Carpentier - con su capacidad de conectarse con el surrealismo francés y proporcionar al público europeo ciertas resonancias exóticas-, así como Guillermo Cabrera Infante - que despliega su fuerza para imponerse literariamente a través de una dimensión política (anti-cubana), y por su capacidad de conectar su obra con métodos experimentales de escritura, sobre todo acuñados por las técnicas del Nouveau roman francés.

Mientras que los autores francófonos e hispanófonos mencionados pueden hacer referencia a cierta recepción, sobre todo en el marco de una clase de lectores bastante especializada y dentro de su capacidad para conectarse con tradiciones literarias europea, fundamentalmente francesas, desde los inicios de la década de 1990, como se sabe, se ha impuesto a nivel mundial un grupo de escritores y escritoras de habla francesa que, aparte de su producción estrictamente literaria, puede hacer gala de una obra ensayística de motivaciones filosóficas que se nutre del contexto de las experiencias en el Caribe. El propósito de los autores y autoras reunidos en torno a las figuras de Patrick Chamoiseau y de Raphaël Confiant, de Martinica, puede entenderse como una variante de la implicación conceptual del multilingüismo en los textos literarios, como un "trato sumamente reflexivo de las normas idiomáticas que tematiza de forma constante la condición fundamental del multilingüismo y la variedad de las lenguas"3

3 "hochreflektierter Umgang mit sprachlichen Normen, der die Grundbedingung der Mehrund Vielsprachigkeit beständig thematisiert" (trad. G.M.). 
(Seiler 2007: 207). El acto de rebelión contra la asimilación cultural, elemento esencial del debate literario en las Antillas, se ve asociado de ese modo no al contenido, sino al nivel estético. El éxito de ese lenguaje literario ha tenido posiblemente consecuencias para el idioma francés normativo, ya que algunos textos escritos en un francés entreverado de oralidad criolla se adentran en un canon literario que se reconstituye y que, en su forma tradicional, conforma la base del francés escrito.

Si prestamos atención a las clásicas instituciones francesas que han demostrado ser decisivas para los procesos de canonización internacional, vemos que un momento clave lo constituye, sin duda, el año 1992, cuando el prestigioso Prix Goncourt, después de más de setenta años, fue otorgado nuevamente a un autor de las Antillas. El último en ganarlo, en 1921, había sido René Maran con su novela Batouala, un acontecimiento que pasó a la historia como la primera vez que un autor declaradamente "negro" recibía un importante premio literario en Francia. Tras una pausa desconcertantemente larga, no adecuada a la realidad de la producción literaria en las Antillas, en 1992 lo gana Patrick Chamoiseau por su novela Texaco. El texto narra una historia que se desarrolla primeramente en la periferia de la capital de Martinica, Fort-de-France, donde ha surgido un barrio marginal de viviendas improvisadas en los antiguos terrenos de la compañía petrolera Texaco, un barrio ilegal que ha de ser arrasado por los buldóceres. La novela se nutre de las diferentes voces de una serie de personajes que dan vida a la historia colonial de Martinica y, de ese modo, representan el entramado de relaciones entre influencias africanas, europeas y asiáticas.

En su ensayo "La littérature française sur le marché mondial des traductions", Gisèle Sapiro escribe que el haber ganado el Prix Goncourt bastó entonces para recibir ofertas de traducción de grandes editoriales, lo cual posiblemente se deba a que algunos libros galardonados con este o con otros premios comparables llegaron a convertirse en bestsellers (Sapiro 2015: 465). Sea como fuere, lo cierto es que, tanto antes como ahora, el Premio Goncourt sigue siendo una referencia obligada para las editoriales estadounidenses. Por regla general, 14 de las 20 obras premiadas consiguen de inmediato una editorial en Estados Unidos (Sapiro 2015: 465). Así ocurrió con obras de Jean Rouaud, Amin Maalouf, Andreï Makine, Patrick Rambaud, Jean-Christophe Ruffin, Marie Ndiaye y Patrick Chamoiseau. Tras recibir el premio en 1992, Texaco fue traducida por Rose-Myriam Réjouis y Val Vinokurov y publicada por Pantheon Books. La novela, según anota Sapiro, fue celebrada por la opinión pública de los Estados Unidos, entre otros por autores como John Updike y el santaluciano Derek Walcott. Se desconocen, sin embargo, las cifras exactas de ediciones y ventas. Si echamos una ojeada a los ganadores del Premio Goncourt en las décadas de 1980 y 1990, comprobamos que, como consecuencia de un interés 
social surgido, sin duda, del poscolonialismo, había una demanda para leer y editar autores y autoras no hexagonales, especialmente africanos y caribeños (Sapiro 2015: 466). Con su novela La nuit sacrée, Tahar Ben Jelloun ganó en 1987 el Premio Goncourt; Patrick Chamoiseau, como ya hemos mencionado, con Texaco, en 1992; Amin Maalouf lo obtuvo con Le rocher de Tanois en 1993; Andreï Makine, por su parte, con Le testament français, en 1995, y Pascale Roze lo recibió por su obra Le chasseur zéro en el año 1996.

Con la entrega a Chamoiseau del Prix Goncourt se inicia una creciente canonización de las literaturas del Caribe. Autores más bien desconocidos hasta ese momento, como Claude McKay, Edward Kamau Brathwaite, Jean Rhys, Jamaica Kincaid, George Lamming, Samuel Selvon, Caryl Phillips, Maryse Condé o Juan Bosch ganaron, en consecuencia, atención internacional. Después del caso de Chamoiseau, ningún otro Premio Goncourt ha ido a parar a las manos de un autor o autora caribeños, pero sí el Prix Médicis, con el que se galardonó en 2009 a un autor haitiano residente en Montreal, Dany Laferrière, por su novela L'enigme du retour, un acontecimiento que puede considerarse igualmente como un momento clave para la producción literaria del Caribe y para su valoración internacional. Esta última novela, con infinidad de referencias intertextuales a la obra de Aimé Césaire Cahier d'un retour au pays natal, pero también a la obra de Naipaul, The Enigma of Arrival, cuenta, desde la perspectiva de una mirada exterior, la historia de una familia que vive a caballo entre Haití y Canadá, una familia en la cual el protagonista, un escritor haitiano en el exilio, regresa a su país de origen, Haití, con motivo del entierro de su padre.

Si preguntásemos en qué tipo de dinámicas se inserta esta creciente canonización, nos tropezamos con una serie de voces críticas que apuntan a la existencia continuada de patrones coloniales: en su estudio The Postcolonial Exotic. Marketing the Margins, Graham Huggan estudia desde una perspectiva sociológica las condiciones materiales de la producción y el consumo de literatura proveniente de contextos coloniales, especialmente la influencia de editoriales e instituciones académicas en la selección, distribución y evaluación de esas obras. Huggan habla de una alterity industry global de la cual los autores y las autoras provenientes de regiones postcoloniales están en vías a menudo de participar en una puesta en escena de su propia marginalidad en la forma ambivalente del exotismo postcolonial ("postcolonial exotic”):

The postcolonial exotic is an effect of commodification, but it is not simply reducible to the cultural logic of consumer capital [. . .] In a sense, it might be seen as a congeries of strategic exoticisms, designed to show the workings of the globalised alterity industry and to repoliticise exotic categories of the cultural other as an unsettling force. [. . .] The language of resistance is entangled, like it or not, in the language of commerce; the anticolonial in the neocolonial; postcolonialism in postcoloniality. What remains - in this 
context at least - is to lay bare the workings of commodification; for the postcolonial exotic is both a form of commodity fetishism and a revelation of the process by which 'exotic' commodities are produced, exchanged, consumed; it is both a mode of consumption and an analysis of consumption (Huggan 2001: 264).

Huggan ve las estrechas relaciones de los autores y autoras postcoloniales con la industria del libro que comercializa la alteridad como un arma de doble filo: por un lado, la expresión de su marginalidad y de su resistencia les concede un especial atractivo para el mercado, pero, por otro lado, esto les abre más posibilidades para el éxito internacional y para el prestigio de importantes premios literarios, lo cual, a su vez, les garantiza un público más amplio y atento, frente al cual los autores y autoras pueden expresar sus propósitos y su postura ambigua en relación con la posición alcanzada: "The postcolonial exotic thus must, in a sense, play the market in order to resist the market, at the same time that resisting the market can be recuperated as playing the market" (Tachtiris 2012: 35s.).

Para Mimi Sheller (2004) los premios literarios europeos constituyen, a pesar de - o precisamente debido a - su prestigio global, vestigios de moldes de pensamiento colonial y de estructuras sociales colonialistas. En el año 1992 -cuando a Chamoiseau le concedieron el Premio Goncourt- se otorgó el Premio Nobel a Derek Walcott, y nueve años después, 2001, a V. S. Naipaul, un premio -el Nobel- que Sheller, en relación con la percepción y la recepción de la literatura no europea, considera un "sello de aprobación de la metrópoli" (Sheller 2003: 185). También Gouhua Zhu y Yonghua Tang ven en el Premio Nobel de Literatura, sobre todo, la expresión de una hegemonía cultural, razón por la cual piden que se termine de una vez la era del Nobel. En la medida en que el jurado del premio prefiere galardonar obras con "atractivo transnacional de carácter transcultural" (Zhu/Tang 2018: 3), solo refuerza teorías filosóficas y estéticas hace tiempo desfasadas acerca de una "naturaleza humana universal" y una "humanidad común" (Zhu/Tang 2018: 3).

En su ensayo "Oraliteracy and Opacity: Resisting Metropolitan Consumption of Caribbean Creole" (2004), Sheller escribe que las culturas occidentales consumen entidades foráneas, es decir, marcadas por sus diferencias raciales ("racially marked bodies”; Sheller 2004: 101), a un nivel visual y metafórico. Esas obras foráneas, exóticas, son descritas por ella como "a kind of spice or condiment to flavour the bland whiteness of mainstream culture" (Sheller 2004: 101; véase también Sheller 2003). También Celia Britton comparte las ideas de Sheller en relación con una especie de consumo de la literatura caribeña. Si antes el consumo se centraba en el azúcar importado de la periferia a la madre patria, ahora ese consumo se concentra en obras literarias que son digeridas por los lectores de la metrópoli sobre la base de la otredad. Por la vía de un tipo de recepción que enfatiza lo directamente sensual de las literaturas del Caribe, se sugiere un 
vínculo directo con el "Otro": "to make the reader feel that s/he is in unmediated contact with the authentic living 'voices' of this exotic culture” (Britton 1996: 18s., cit. según Sheller 2004: 104). Ese exotismo percibido en Europa o Estados Unidos puede reflejarse lo mismo a nivel idiomático que a nivel del contenido. Lo problemático de esta forma de exotización es, sobre todo, que el grado de resistencia que el texto ajeno opone al entendimiento general acaparador, descrita por algunos teóricos de la categoría de Édouard Glissant como una "opacidad" entendida en sentido positivo, queda derogada con la actitud consumista de la recepción en Occidente (véase Britton 1996: 18s., cit. según Sheller 2004: 104).

Sin embargo, A. James Arnold ve también un motivo para ello en las propias obras. En su caso, el reproche se dirige especialmente a los autores y autoras antillanos que se mercantilizan en favor de los lectores europeos, por ejemplo, con la decisión de escribir en francés: las novelas, dice Arnold, aparecen a menudo "formally designed to appeal to the neo-colonialist desire of the European narratee to see in the West Indies a timeless society, forever playing out the same plot” (Arnold 1998: 47; cit. según Tachtiris 2012: 92). Los autores que abogan realmente por la independencia de sus islas, contribuirían de ese modo, con sus obras, a una "estética de la dependencia” (Arnold 1998: 47; cit. según Tachtiris 2012: 92).

La relación de dependencia entre los autores y autoras no europeos y los lectores y lectoras europeos o internacionales se refleja también en la selección de las casas editoriales. Es cierto que existen editoriales caribeñas como Ibis Rouge, en Guadalupe, pero el alcance de las editoriales pequeñas es muy limitado y no puede garantizarle al autor el acceso al mercado internacional o se lo garantiza, en todo caso, de un modo restringido (Dumontet 2000: 164). No asombra, por ello, que las diez novelas de Patrick Chamoiseau hayan sido publicadas por Gallimard, que, como sabemos, es una de las editoriales más influyentes de Francia (Tachtiris 2012: 91).

Si observamos ahora la circulación de la novela premiada de Chamoiseau, Texaco, más allá del ámbito de habla francesa y prestamos atención a la cubierta de la traducción alemana publicada por Piper Verlag, se pone claramente de manifiesto que la estrategia de marketing apuesta por el énfasis en el exotismo caribeño y las reminiscencias coloniales: lo que vemos en la portada es el cuadro de una mujer de piel oscura que está sentada y cuya tez se ve realzada por el blanco radiante de su vestido. Le cubre la cabeza un pañuelo igualmente blanco. El vestido, cuyo aspecto se asemeja más bien al de una sábana ceñida en la cintura, cae desde los hombros y deja el torso y sus senos desnudos expuestos a las miradas del espectador. El cuadro reproducido en la portada es una obra de la artista francesa Marie-Guillemine Benoist y no solo ha servido exclusivamente como cubierta de la obra de Chamoiseau. El mismo año que 
apareció la traducción de Texaco, se publicó también en la editorial S. Fischer la novela Die Mohrin [La mora], de Lukas Hartmann. La portada era la misma, con la única diferencia que los pechos de la mujer en la portada de La mora estaban completamente cubiertos. Pero hay más: ya en 1988 la editorial Gallimard había empleado el mismo cuadro para ilustrar la portada de la edición folio de Moi, Tituba, sorcière . . . Noire de Salem, la novela de Maryse Condé. Los pechos al desnudo de la mujer de piel oscura en relación con las novelas de Chamoiseau y de Condé, ambos autores del Caribe, confiere a sus obras rasgos exotizantes. Una portada de esta índole transmite de forma condensada la mirada racista, sexista y cosificadora del colonialismo europeo y que, en el contexto literario, se asocia a una apropiación de lo ajeno con fines de consumo.

Es justo ese ángulo de visión el que, en el caso de la obra de Chamoiseausolo por mantener el mismo ejemplo-, se proyecta sobre su obra, y ello ocurre a pesar de que la novela, por la orientación de su contenido, tiene fines completamente opuestos. Texaco es todo menos un producto de consumo fácil, su autor desarrolla un lenguaje altamente elaborado, situado entre el créol y el francés normativo, en una deliciosa amalgama de neologismos y asociaciones verbales, de giros y estructuras idiomáticos orales de esa lengua criolla y originalísimas formas de escritura, toda una retórica créol con diferentes variantes normativas del francés normal y del francés normativo, desde el más puro lenguaje literario hasta otras formas arcaicas del habla ${ }^{4}$. Falk Seiler enfatiza la fuerza subversiva de la norma en este trabajo con la lengua. Sobre esto escribe que se trata de una "una doble estrategia emancipadora de Chamoiseau, la de no contraponer solamente a una norma literaria fijada la flexibilidad de una literatura oral impregnada por el créol, sino en su voluntad de seguir el rastro a lo subversivo en la propia tradición literaria de Francia e intentar conectar con ella con todos los medios disponibles"5 (Seiler 2007: 211).

\footnotetext{
4 Véase el detallado análisis estilístico del lenguaje de Chamoiseau que Danielle Dumontet antepone a su crítica a la traducción de Texaco: "Patrick Chamoiseau n'utilise pas forcément le créole, la langue de l'oral, dans ses dialogues mais surtout et avant tout dans ses séquences narratives, dans lesquelles il mêle les citations lexicales ou phrases en créole, les créations lexicales ('déparler', 'malement', etc.), les composés ('son manger-macadam', etc.), les constructions nominales (sans article), les marqueurs créoles ('une charge de temps', 'toutes qualités de paroles' ... ), les constructions verbales particulières telles que 'elle prit-courir', les interrogations et assertions avec marqueurs telles que 'han' ou 'ho', les choix graphiques, toujours symboliques tels que les 'céhêresses' (CRS), les 'achélèmes' (HLM), les onomatopées créoles ou interjections tels 'tomber flip', 'comprendre hak', etc., et enfin les figures du discours où transparaît une certaine rhétorique créole” (2000: 167).

5 "emanzipatorische[] Doppelstrategie Chamoiseaus, nicht lediglich einer bestimmten literarisch fixierten Norm die Flexibilität einer kreolisch geprägten Oraliture entgegenzustellen, sondern das
} 
La discrepancia entre un manejo sumamente reflexivo del medio lenguaje, un manejo que, en el contexto de un entorno histórico específico, genera nuevas normas lingüísticas del francés, y la estrategia de mercado transmitida en la portada, la cual promete los mismos digeribles clichés exotizantes y archiconocidos, puede haber sido uno de los motivos para que la traducción alemana de Texaco fuera un fracaso en las librerías: las cifras de ventas se movieron entre los 2000 y los 3000 ejemplares (Dumontet 2000: 172). Dumontet indica, además, que ese fracaso tuvo efectos negativos en el mercado del libro para la posterior publicación de obras de autores y autoras caribeños de habla francesa, ya que la conclusión del ramo fue que en Alemania no había un público para tales problemáticas (Dumontet 2000: 172).

El autor Dany Laferrière, ya mencionado como ganador del Prix Médicis en 2009, es otro ejemplo - aunque distinto- de ese "exotismo postcolonial" como estrategia de publicación en el mercado global del libro. En su caso se pone de manifiesto el modo en que la puesta en escena subversiva de las estéticas y las prácticas colonialistas y exotizantes en la circulación y comercialización puede desplegar una dialéctica de reflexión crítica y de consumo acaparador. Las obras del autor natural de Haití, emigrado a Montreal en 1976 por motivos políticos, ha alcanzado entre tanto 31 libros publicados y está, sobre todo en sus comienzos, marcada por un tratamiento muy ofensivo de los motivos colonialistas y racistas: la primera novela de Laferrière, con el provocador título de Comment faire l'amour avec un nègre sans se fatiguer, apareció en 1985 en Quebec y proporcionó a su autor, de golpe, una gran fama entre los lectores canadienses de habla francesa. Al mismo tiempo, Laferrière se distancia constantemente de la coacción de ser encasillado como autor postcolonial de temas (post)coloniales, como se pone claramente de manifiesto en la traducción al inglés de su quinta novela: Cette grenade dans la main du jeune Nègre est-elle une arme ou un fruit? (Montreal 1993): Why Must a Black Writer Write About Sex? (Toronto 1994).

Sin embargo, en lo relativo a otros mercados del libro, como el de Francia, Alemania o Haití, su obra solo ha podido conquistarlos de manera notoriamente

\footnotetext{
Subversive in der literarischen Tradition Frankreichs selbst aufzuspüren und mit allen ihm zu Gebote stehenden Mitteln daran anzuknüpfen” (trad. G.M.). El propio Chamoiseau ha descrito su propósito en una carta a sus traductores dispersos por el mundo, los cuales se pusieron a trabajar en las versiones a sus respectivos idiomas a raíz del otorgamiento del Premio Goncourt a la novela Texaco: "J'essaie de faire en sorte que la langue perde de son orgueilleuse certitude, de son académisme formel, je veux qu'on la sente tremblante, disponible pour toutes les autres langues du monde; qu'elle ne donne plus l'impression d'être la seule a pouvoir dire le monde mais qu'on la sente relativisée, problématisée, informée de la splendeur possible des autres langues placées désormais sur le même plan" (cit. según Seiler 2007: 211, quien, a su vez, menciona a Micaux 1997: 61 como fuente).
} 
más lenta, mientras que en Estados Unidos su escritura solo tuvo, al principio, muy escasa resonancia (Tachtiris 2012: 59). En Francia, sus libros se publican desde 1997 en la pequeña editorial Le Serpent à Plumes, y desde 2005 en la prestigiosa editorial Grasset. En el año 2007 apareció una nueva versión ampliada de Le goût des jeunes filles en Gallimard (Tachtiris 2012: 92, n. 34). En el caso de las distintas vías de circulación a nivel global, hemos de vérnoslas con dinámicas muy diversas; vemos, por ejemplo, cómo para que un libro logre imponerse en el mercado internacional, resulta decisivo que, si se trata de un autor de habla francesa en una editorial canadiense, su obra sea publicada también por una editorial especializada parisina o por una de las prestigiosas casas como Grasset o Gallimard. Además, tal como señala Corine Elizabeth Tachtiris, no existe eso que llaman un "lector globalizado", sino que los contextos de recepción difieren claramente en los distintos casos. Más aún:

What this means in terms of the circulation of world literature is that a book is not guaranteed a similar reception in different markets because not only is the context not the same, neither is the product, the book, itself. Laferrière has been particularly successful in a variety of markets because of the way that he and the other agents who rewrite his work (in the Lefevrian sense) have been shrewd about transacting in different forms of currency in different markets (Tachtiris 2012: 52s.).

Mientras que en el contexto francófono de Quebec tuvo una enorme resonancia, desde la primera novela, la estridente puesta en escena del inmigrante negro, la recepción en Haití ha tenido lugar a partir de la tercera obra, L'odeur du café, la primera que cuenta con una referencia clara al país caribeño. En Francia, a su vez, los autores de la créolité reunidos en torno a las figuras de Raphaël Confiant y a Patrick Chamoiseau determinaban los discursos en relación con la literatura de habla francesa con referencias al Caribe, de modo que otras obras de Laferrière consiguieron irse imponiendo lentamente hacia finales de los años 90, si bien el autor mismo las reescribió o amplió en parte, a fin de adaptarlas a los discursos predominantes en el mercado del

\footnotetext{
6 Véase también la crítica de Sarah Brouillette a la idea de un "global market reader" más bien simple, aun cuando ese lector se diseñe en obras del exotismo postcolonial, a menudo, como lector implícito: "[. . .] strategic exoticism is not something a writer deploys to teach a reader about the errors in her conceptions about other cultures, much though it depends upon a construction of a figure in need of such instruction. Instead, it indicates a set of textual strategies that communicates at all because the author and the actual reader likely share assumptions about the way culture operates, and concur in their desire to exempt themselves from certain undesirable practices" (2007: 43).
} 
libro en Francia ${ }^{7}$. A partir de 2013 -es decir, después de que, en 2005, el autor de raíces haitianas diera el gran salto a las editoriales francesas de prestigio-, se le editó también en Alemania. Desde entonces, tres de sus obras han sido publicadas en la pequeña editorial de Heidelberg Das Wunderhorn, en traducción de Beate Thill ${ }^{8}$. La última, en el año 2017, fue su novela debut, que apareció bajo el título de Die Kunst, einen Schwarzen zu lieben ohne zu ermüden [El arte de amar a un negro sin cansarse]. Este texto, con más de treinta años de antigüedad y al que le importan un comino las normas de la political correctness, fue vendido a los lectores de habla alemana como un aporte original a los actuales debates en torno a los estudios culturales: “[. . .] la objeción más sexy a la programática de una Critical Whiteness y el libro del momento por antonomasia"9.

Y por último quisiera referirme al ejemplo más actual imaginable en este contexto, el de Maryse Condé: en 2018, a raíz del escándalo en torno al comité del Premio Nobel de Literatura, se otorgó el Premio Nobel alternativo, convocado en su lugar por los libreros suecos, a esta autora tan fuertemente asociada a la confrontación con el colonialismo. Maryse Condé, autora de numerosas novelas, cuentos, piezas teatrales, libros infantiles y ensayos, nació en la isla de Guadalupe, vivió en París y en varios países de África, y más tarde dio clases en la Sorbona y en la Universidad de Columbia. En la actualidad, su vida alterna entre Nueva York y Guadalupe. Agradeció la distinción en un mensaje transmitido por vídeoconferencia. Le alegraba el reconocimiento, dijo, "but please allow me to share it with my family, my friends and above all the people of Guadeloupe, who will be thrilled and touched seeing me receive this prize”. Añadió. "We are such a small country, only mentioned when there are hurricanes or earthquakes and things like that. Now we are so happy to be recognised for something else" (The Guardian, 12/10/2018). Tanto en lo que atañe a los temas de sus novelas como en relación con su biografía, Maryse Condé encarna como quizá ninguna otra autora o autor la disolución de la idea vinculada al centro y

7 Me refiero aquí a Corine Elizabeth Tachtiris, quien, en su disertación Branding World Literature: The Global Circulation of Authors in Translation (2012: 32-103), analiza con todo detalle la recepción internacional de las obras de Laferrière.

8 Las dos, la editorial y la traductora, han hecho muchos méritos en Alemania en lo relativo, sobre todo, a la difusión de las literaturas del Caribe. Beate Thill ha traducido para Wunderhorn, entre otros, a Édouard Glissant, o algunas obras de Patrick Chamoiseau, como el ensayo crítico Migranten, de 2017.

9 Esta cita, tomada de la reseña publicada por Brigitte Werneburg en el periódico tageszeitung, es presentada en la página web de la editorial Das Wunderhorn en un lugar destacado de la larga lista dedicada al dossier de prensa. Véase <https://www.wunderhorn.de/?buecher= die-kunst-einen-schwarzen-zu-lieben-ohne-zu-ermueden>. 
la periferia; al mismo tiempo, representa el indisoluble conflicto que surge de la circunstancia de que los centros de denominación sigan estando en Occidente, en ciudades como, en este caso, París y Estocolmo.

\section{Conclusión}

Vemos que, en lo que respecta a la práctica editorial y de canonización, especialmente en Francia, surge por un lado, muy lentamente, un nuevo movimiento: también los autores y las autoras del Caribe van ocupando, aunque a paso vacilante, su lugar en las instituciones decisivas de Francia u Occidente, y ello comienza con el otorgamiento del premio Goncourt a Chamoiseau en el año 1992. Por otra parte, vemos también cómo en la práctica de la concesión de premios y de la a ella asociada práctica de publicaciones, como es el caso de Gallimard y de otras editoriales con una gran actividad internacional, se sigue actuando según la lógica de centro-periferia que tiene una larga tradición y a la que no se va a renunciar con la inclusión creciente de bienes culturales provenientes del Caribe. Muchas veces esa dinámica puede entenderse como una dinámica de consumo en la que se da continuidad a las antiguas implicaciones coloniales. El exotismo postcolonial, como en el caso de Laferrière, aparece aquí como una estrategia de publicación que impulsa esa dinámica de consumo a través de la crítica a las implicaciones coloniales, mientras que, al mismo tiempo, esa postura crítica solo puede lograr ser escuchada gracias a su presencia en el mercado global del libro. No podemos, sin embargo, pasar por alto que son precisamente los autores y autoras que representan, desde el punto de vista de sus contenidos, una efectiva estética caribeña, una autonomía y singularidad cultural e idiomática del Caribe, los que no pocas veces contribuyen a la fijación de esas relaciones de dependencia dentro de las dinámicas de los procesos de traducción cultural en la práctica de la difusión y publicación de la literatura. De ello tenemos un buen ejemplo, como hemos visto, en Patrick Chamoiseau. Por otra parte, además, a la hora de introducir la literatura del Caribe en otros mercados del libro, se consideran distintas estrategias editoriales adaptadas a los contextos de recepción específicos de los distintos lectores; estrategias que no siempre dan buenos resultados, como el de la cubierta transmisora de exotismo colonial como esquema de comercialización en el caso de Texaco, la premiada novela de Chamoiseau.

Cabe esperar o examinar con más detalle el modo en que la influencia de las grandes casas editoriales tendrá efecto en la presencia de las literaturas del Caribe en el mercado global del libro. El autor mencionado al principio, André 
Schiffrin, antiguo editor de Pantheon Books, comentó ampliamente, a raíz de su salida de la editorial, las controversias condicionadas por su labor en ese ramo. Controversias entre su posición y las posiciones en Random House -el Knopf Publishing Group, incluido Pantheon, pertenecía desde 1961 a Random House-, así como las dinámicas que surgieron a raíz de que el grupo fuera absorbido por Bertelsmann en 1998. Schiffrin critica que los representantes de la multinacional Bertelsmann no estuvieran ya interesados en representar a autores y autoras, sino más bien a colocar en el mercado determinados libros aislados, una práctica llevada adelante por editores y editoras que ya no tenían tiempo para leer y apenas conocían los contenidos. A la par de este proceso, según me parece, veremos concretarse un aumento de los diseños de portada exotizantes en los títulos de libros sobre el Caribe, una observación que habrá que verificar y especificar. En qué medida la homogenización criticada y lamentada por Schiffrin y la orientación al beneficio por parte de las casa editoriales condicionarán en el futuro la selección y canonización de las literaturas del Caribe, precisamente en el mundo de habla inglesa, es algo a lo que debemos estar atentos.

\section{Bibliografía}

“Alternative Nobel literature prize goes to Maryse Condé”. En: The Guardian, 12/10/2018, <https://www.theguardian.com/books/2018/oct/12/alternative-nobel-literature-prizemaryse-conde-new-academy-prize> (última visita: 25/03/2020).

“Literarisches Sextett”, Mesa redonda en el marco de las Kölner Gespräche zur Weltliteratur 2018. Participantes: Florian Borchmeyer (Schaubühne Berlín), Andreas Breitenstein (Neue Zürcher Zeitung), Jo Lendle (editorial Hanser), Sandra Richter (Archivo de la Literatura Alemana en Marbach), Andreas Rötzer (editorial Matthes \& Seitz), Uljana Wolf (Autora); Moderación: Benjamin Loy. Videoconferencia online, <https://youtu.be/8_4SoJPiH4> (última visita: 17/02/2020).

“Literaturbetrieb global”, Mesa redonda en el marco de las Kölner Gespräche zur Weltliteratur 2016. Participantes: Heinrich von Berenberg, Anita Djafari (Litprom), ljoma Mangold (DIE ZEIT), Thomas Sparr (Suhrkamp Verlag), Nicole Witt (Agencia literaria Witt Mertin); Moderación: Benjamin Loy. Videoconferencia online, <https://www.youtube.com/watch?

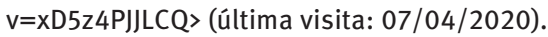

Arnold, A. James (1998): “Créolité: Cultural Nation-Building or Cultural Dependence?”. En: D’haen, Theo (ed.): (Un)writing Empire. Atlanta: Rodopi, pp. 37-48. (Cross/Cultures: Readings in the Post/Colonial Literatures in English 30).

Barbery, Muriel et al. (2007): “Pour une 'littérature monde' en français”. En: Le Monde, 15/03/ 2007 (actualizado el 03/02/2011), <https://www.lemonde.fr/livres/article/2007/03/15/ des-ecrivains-plaident-pour-un-roman-en-francais-ouvert-sur-le-monde_883572_3260. html> (última visita: 28/01/2020). 
Before Columbus Foundation: 〈http://www.beforecolumbusfoundation.com/about/> (última visita: 25/03/2020).

Britton, Celia (1996): "Eating their words: The consumption of French Caribbean literature". En: Association for the Study of Caribbean and African Literature in French (ASCALF) Yearbook 1, pp. 5-23.

Brouillette, Sarah (2007): Postcolonial Writers in the Global Literary Marketplace. Nueva York: Palgrave Macmillan.

Dumontet, Danielle (2000): "Possibilités et limites des transferts culturels: le cas des romans La Reine Soleil levée de Gérard Étienne et Texaco de Patrick Chamoiseau”. En: TTR: Traduction, terminologie, rédaction 13/2 (Dossier "Les Antilles en traduction/The Caribbean in Translation", ed. by Anne Malena), pp. 149-178.

Huggan, Graham (2001): The Postcolonial Exotic: Marketing the Margins. Londres: Routledge. Micaux, Wandrille (1997): “Le lexique des 'marqueurs de parole’ antillais Patrick Chamoiseau et Raphaël Confiant”. En: Études Creoles 20/2, pp. 59-69.

Müller, Gesine (2017a): "El debate sobre la literatura mundial y sus dimensiones editoriales: la región del Caribe a modo de ejemplo". En: Revista chilena 96, pp. 67-85.

Müller, Gesine (2017b): “Karibische Literaturen zwischen 'Weltliteratur' und 'Literaturen der Welt”. En: Lendemains XLII/168 (Dossier “Les Antilles et les littératures du monde”, ed. de Ottmar Ette, Gesine Müller), pp. 73-84.

Müller, Gesine/Neumann, Birgit (en prensa): "Weltliteraturen in den Amerikas. Die Karibik (englisch, französisch, spanisch)”. En: Borsò, Vittoria (ed.): Handbuch Weltliteratur. Berlín: De Gruyter.

Sapiro, Gisèle (2017): "The Role of Publishers in the Making of World Literature: The Case of Gallimard". En: Letteratura e Letterature 11, pp. 81-93.

Sapiro, Gisèle (2016): "How Do Literary Works Cross Borders (or Not)? A Sociological Approach to World Literature”. En: Journal of World Literature 1, pp. 81-96.

Sapiro, Gisèle (2015): “La littérature française sur le marché mondial des traductions". En: McDonald, Christie/Rubin Suleiman, Susan (eds.): French Global. Une nouvelle perspective sur l'histoire littéraire. París: Classiques Garnier, pp. 447-483.

Sapiro, Gisèle/Pacouret, Jérôme/Picaud, Myrtille (2015): "Transformations des champs de production culturelle à l'ère de la mondialisation”. En: Actes de la recherche en sciences sociales 206/207, pp. 4-13.

Schiffrin, Andre (2000): The Business of Books: How International Conglomerates Took Over Publishing and Changed the Way We Read. Londres/Nueva York: Verso.

Seiler, Falk (2007): “Literarisches Schreiben auf den Antillen im Spannungsfeld zwischen Französisch und Kreolisch: Patrick Chamoiseau”. En: Laferl, Christopher F./Pöll, Bernhard (eds.): Amerika und die Norm: Literatursprache als Modell? Tubinga: Niemeyer, pp. 207-224.

Sheller, Mimi (2004): “Oraliteracy and Textual Opacity: Resisting Metropolitan Consumption of Caribbean Creole”. En: Academic Journal Language and Intercultural Communication 4/1-2, pp. 100-108.

Sheller, Mimi (2003): Consuming the Caribbean: From Arawaks to Zombies. Londres: Routledge.

Steiner, Ann (2011): “World Literature and the Book”. En: D’haen, Theo/Damrosch, David/ Kadi, Djelal (eds.): The Routledge Companion to World Literature. Londres/Nueva York: Routledge, pp. 316-324. 
Tachtiris, Corine Elizabeth (2012): Branding World Literature: The Global Circulation of Authors in Translation. Dissertation. University of Michigan. <https://deepblue.lib.umich.edu/bits tream/handle/2027.42/93838/tachtco_2.pdf?sequence=1\&isAllowed=y> (última visita: 25/03/2020).

Verlag Das Wunderhorn: <https://www.wunderhorn.de/?buecher=die-kunst-einen-schwarzenzu-lieben-ohne-zu-ermueden> (última visita: 25/03/2020).

Zhu, Gouhua/Tang, Yonghua (2018): "The End of the Nobel Era and the Reconstruction of the World Republic of Letters”. En: CLCWeb: Comparative Literature and Culture 20/7, 〈https://doi.org/10.7771/1481-4374.3329> (última visita: 25/03/2020). 
